\title{
NIVELES DE HIDROPERÓXIDOS DE PROTEÍNAS Y DE GRUPOS CARBONILOS EN ÓRGANOS DE COBAYOS NATIVOS DE ALTURA (HUANCAVELICA, $3660 \mathrm{M}$ )
}

\author{
Levels of protein hydroperoxides and carbonyl groups in guinea pigs native of high altitudes \\ (Huancavelica, $3660 \mathrm{~m}$ ) \\ Roxana Huayta ${ }^{1}$, Haydée Zúñiga ${ }^{1}$, Cynthia Esquerre ${ }^{2}$, Luz Hernández ${ }^{2}$ y Elizabeth Carranza ${ }^{1}$ \\ ${ }^{1}$ Instituto Nacional de Biología Andina, Facultad de Medicina. ${ }^{2}$ Facultad de Farmacia y Bioquímica. UNMSM
}

\section{RESUMEN}

Se evaluó la influencia de la hipoxia hipobárica sobre la oxidación de proteínas en pulmónes, corazón, hígado, riñónes y testículos de cobayos nativos de altura (Huancavelica, $3660 \mathrm{~m}$ ) en comparación con los de cobayos oriundos de nivel del mar (Lima, $150 \mathrm{~m}$ ). Se determinó la concentración de hidroperóxidos de proteínas (POOH) y de grupos carbonilo (GC) como marcadores de oxidación proteica, así como la concentración de tioles totales (TT), potentes agentes reductores que actúan como antioxidantes en vivo. Los resultados mostraron menores concentraciones de grupos carbonilos en testículos de cobayos de altura, comparados con los de nivel del mar. También se encontró menor concentración de POOH en corazón de cobayos de altura, respecto a los de nivel del mar. La concentración de TT, también fue menor en corazón y en pulmónes de cobayos de altura que en los de nivel del mar. Contrariamente a estos resultados, se hallaron elevadas concentraciones de hidroperóxidos proteicos en hígado y testículos, y de tioles totales únicamente en riñónes de cobayos de altura. Estos resultados indican que la hipoxia hipobárica influye en forma selectiva en la oxidación de proteínas y en la respuesta antioxidante endogéna no enzimática.

Palabras clave: Oxidación de proteínas, tioles totales, cobayos, hipoxia hipobárica.

\section{SUMMARY}

The influence of hypobaric hypoxia on protein oxidation in lungs, heart, liver, kidneys and testicles of high altitude native guinea pigs (Huancavelica, $3660 \mathrm{~m}$ ) in comparison to sea level (Lima, $150 \mathrm{~m}$ ) native guinea pigs was evaluated. The concentration of protein hydroperoxides $(\mathrm{POOH})$ and carbonyl groups $(\mathrm{GC})$ as markers of protein oxidation, as well as total thiols (TT) concentration, powerful reducing agents that act as live antioxidants were determined. The results showed low concentration of GC in high altitude guinea pig testicles, as compared to what was found at sea level. POOH low concentration was also found in high altitude guinea pig heart with respect to that found at sea level. TT concentration was lower in high altitude heart and lungs in relation to the concentrations found at sea level. Contrary to these results, higher concentrations of $\mathrm{POOH}$ in high altitude guinea pig liver and testicles were found, while increase in TT is observed only in the high altitude guinea pig kidneys. These results show that the hypobaric hypoxia selectively influences on the oxidation of proteins and on endogenous nonenzymatic antioxidant response.

Keywords: Oxidation, guinea pigs, height, protein, hypobaric hypoxia.

\section{INTRODUCCIÓN}

$\mathrm{E}$ videncias recientes demuestran la existencia de una asociación entre estrés oxidativo e hipoxia, así se ha encontrado un incremento de sustancias reactivas derivadas del oxígeno en modelos de hipoxia/reperfusión, ejercicio físico agotador, hipoxia simulada en cámara hipobárica ${ }^{(1)}$, en el ascenso a grandes alturas ${ }^{(2)}$, así como también en residentes de las grandes alturas ${ }^{(3)}$. En las poblaciones que viven en las grandes alturas se pueden incluir además de la hipoxia, otras fuentes de estrés oxidativo, como el aumento a la luz ultravioleta, la exposición al frío, el aumento de ejercicio, factores dietéticos y la estimulación del sistema nervioso simpático ${ }^{(1)}$.

El oxígeno, elemento imprescindible para los organismos aerobios para producir energía, puede también alterar los sistemas biológicos a través de una producción incontrolada de radicales libres derivados del oxígeno (EROS) de origen endógeno y/o exógeno, dañando moléculas biológicas, alterando procesos celulares y desencadenando trastornos en el metabolismo celular.

Existe evidencia que indica que el mantenimiento del estado redox de la proteína es fundamental para la 
función célular, por lo tanto, los cambios estructurales en las proteínas son considerados como uno de los mecanismos moleculares que conduce, por ejemplo, a la disfunción endotelial ${ }^{(4)}$.

Está bien establecido que la exposición de proteínas a especies reactivas del oxígeno (ROS) puede alterar la estructura química y física de la molécula, causando oxidación consiguiente de los grupos laterales, escisión de proteínas, fragmentación del esqueleto proteico, entrecruzamiento, pérdida del plegamiento, y formación de nuevos grupos reactivos. Esto último, incluye oxidación de derivados de los residuos aminoacil hidrofóbicos, hidroxi e hiperoxi $(\mathrm{POOH})$, carbonilación de proteínas $(\mathrm{GC})$, formación de nitrotirosina (NT) y muchos otros. Los cambios conformacionales que resultan de este complejo de reacciones conllevan a la disminución o pérdida de la función biológica de la proteína ${ }^{(5,6)}$. Si bien la vida en las grandes alturas está acompañada de un ambiente hipóxico, el propio organismo desarrolla estrategias fisiológicas y bioquímicas de adaptación, que si no fueran del todo eficientes podrían desencadenar un incremento del estrés oxidativo, conduciendo a un daño sobre la estructura biológica proteica, daño que puede ser identificado a través de marcadores específicos de oxidación.

Estudios sobre el efecto de la hipoxia de las grandes alturas sobre el metabolismo oxidativo proteico en órganos de mamíferos son escasos, por lo que se ha considerado de interés medir los niveles tisulares de dos marcadores de oxidación de proteínas: hidroperóxidos de proteínas $(\mathrm{POOH})$ y grupos carbonilos (GC), así como el contenido de tioles totales (TT) como indicador de defensa antioxidante, en órganos de cobayos nativos de altura.

\section{MATERIAL Y MÉTODOS}

\section{Diseño experimental}

Se emplearon 10 cobayos (Cavia porcellus) adultos, machos nacidos y criados en la altura (Huancavelica, $3660 \mathrm{~m})$ y alimentados ad-libitum con chala de maíz (Zea mays L), hasta alcanzar un peso promedio de 500 g. El grupo considerado como control fueron 10 cobayos machos adultos nacidos y criados a nivel del mar (NM) (Lima, $150 \mathrm{~m}$ ).

Los animales de cada grupo fueron sacrificados en su lugar de origen. Las pruebas analíticas fueron llevadas a cabo en el Instituto Nacional de Biología Andina en Lima.
Veinticuatro horas antes de ser sacrificados los animales fueron dejados en ayunas. Los cobayos fueron sacrificados por dislocación cervical, y el hígado, corazón, riñónes, pulmónes y testículos fueron extraídos rápidamente, lavados con cloruro de sodio helado $0,9 \%$ a $4^{\circ} \mathrm{C}$, secados en papel de filtro e inmediatamente colocados en bolsas de polietileno previamente identificadas, transportados en nitrógeno líquido y se conservó a $-40^{\circ} \mathrm{C}$ hasta el análisis.

Para el análisis se pesó aproximadamente $1 \mathrm{~g}$ de cada órgano, el cual fue homogeneizado con buffer fosfato salino (BPS) $50 \mathrm{mmol}, \mathrm{pH} 7,0$ a $4^{\circ} \mathrm{C}$, por un tiempo no mayor a $3 \mathrm{~min}$. Los respectivos homogeneizados fueron usados para realizar las determinaciones de hidróxidos de proteínas, grupos carbonilos, tioles totales y proteínas.

\section{Determinaciones}

La concentración de hidroperóxidos de proteínas se midió por el método descrito por Nourooz y col (7). El ensayo se basa en la oxidación de $\mathrm{Fe}^{2+}$ por peróxidos, en la presencia del colorante naranja de xilenol, que forma un complejo coloreado con el $\mathrm{Fe}^{3+}$ generado. El complejo de Fe-XO se puede medir por espectrofotometría a 560 ๆm.

Procedimiento: Se tomaron $300 \mu \mathrm{L}$ de homogeneizado, se les adicionó $1500 \mu \mathrm{L}$ de ácido perclórico $0,2 \mathrm{M}$, se llevó a baño de hielo por 5 minutos, luego se procedió a centrifugar la muestra a $6500 \mathrm{rpm}$. El precipitado fue lavado con $1275 \mu \mathrm{L}$ de una solución clorofórmica que contiene BHT 4 $\mathrm{mM}$, se centrifugó y se descartó el sobrenadante. Las proteínas precipitadas se disolvieron en $1650 \mu \mathrm{L}$ de hidrocloruro de guanidina $6 \mathrm{M}$.

Se removieron $1500 \mu \mathrm{L}$ de la fase acuosa, y se adicionaron en este orden: $60 \mu \mathrm{L}$ ácido perclórico 0,5 $\mathrm{M} ; 37,5 \mu \mathrm{L}$ de xilenol de naranja $5 \mathrm{mM} ; 37,5 \mathrm{~mL}$ de agua bidestilada; $15 \mu \mathrm{L}$ de solución ferrosa $\left(\mathrm{Fe}^{+2}\right)$. Se incubó por 60 minutos a temperatura estable y oscuridad, y se leyó a 560 ๆm frente a un blanco que contenía hidrocloruro de guanidina $6 \mathrm{M}$ en lugar de la muestra de homogeneizado.

Para los cálculos se trabajó con la diferencia de la absorbancia entre la muestra y su respectivo blanco ( $\Delta$ Abs), usando el coeficiente de extinción molar del complejo formado $\left(\varepsilon=37 \times 10^{4} \mathrm{M}^{-1} \mathrm{~cm}^{-1}\right)$. Los resultados se expresaron como ๆmoles de $\mathrm{P}-\mathrm{OOH} / \mathrm{mg}$ de proteína.

El contenido de GC se determinó según el método descrito por Levine y col. ${ }^{(8)}$, basado en la formación de 2,4-dinitrofenilhidrazona, producto de la reacción 
entre los grupos carbonílicos de las proteínas oxidadas y el reactivo 2,4-dinitrofenilhidrazina $(2,4-\mathrm{DNPH})$ en medio ácido.

La concentración de tioles totales se determinó usando 5,5-ditio-bis (2-ácido nitro-benzoico) (2DTNB) tal como lo describe Cakatay ${ }^{(9)}$.

Procedimiento: Se tomaron $50 \mu \mathrm{L}$ de homogeneizado, se adicionaron $500 \mu \mathrm{L}$ de buffer TRIS o,2 M, pH 8,2 y $100 \mu \mathrm{L}$ de 2 -DTNB o,o1M, se adicionaron $1000 \mu \mathrm{L}$ de metanol absoluto, se preparó un blanco de reactivo ( $\sin$ muestra), se dejó reposar por 15 minutos; se centrifugó en frío a $6000 \mathrm{rpm}$.

El sobrenadante fue leído en un espectrofotómetro a $412 \eta \mathrm{m}$. Para los cálculos se utilizó el coeficiente de extinción molar del producto formado en la reacción (2-nitro-5-tiobenzoato, $\varepsilon_{\mathrm{NTB}}=1,31 \times 10^{4} \mathrm{M}^{-1} \mathrm{~cm}^{-1}$ ). Los resultados fueron expresados en $\eta \mathrm{mol}$ de tioles/mg de proteína.

Para la determinación de proteínas se utilizó el método de Bradford, que se fundamenta en la formación de un complejo del reactivo azul coomasie y las proteínas en solución, causando un cambio en el máximo de absorción de 595 ๆm.

\section{Análisis Estadístico}

Para el análisis estadístico de datos se utilizó el programa estadístico SPSS 18, Los resultados obtenidos de los grupos estudiados, tanto a nivel del mar como altura, se expresan como mediasy desviaciones estándar (SD). Para verificar la significancia de las diferencias entre las medias aritméticas de cada parámetro se empleó la prueba de $t$ de Student, y se evaluó el grado de asociación mediante el coeficiente de correlación de Pearson. Se considera estadísticamente significativo a todo resultado cuyo valor asociado de $p$ sea $\leq$ que 0,05

\section{RESULTADOS}

En la figura 1 se presentan los resultados de los niveles medios de $\mathrm{POOH}$, según órgano. El nivel medio ( $\eta$ moles/mg proteína) encontrado en corazón de cobayo de altura $(2,5 \pm 2,0)$ fue significativamente menor $(p=o, o 3)$ que su similar del nivel del mar $(4,0$ $\pm 2,0$ ), mientras que los valores medios de hígado $(5,19 \pm 1,6$ vs $10,6 \pm 4,8)$ y testículos $(1,5 \pm 0,5$ vs $4,5 \pm$ $0,6)$ fueron significativamente mayores en cobayos de la altura que a nivel del mar $(p=o, o o o)$. Asimismo, no se encontraron diferencias significativas de este marcador de oxidación en pulmones $(6,1 \pm 1,6$ vs 7,2 \pm $0,8)$ y riñones $(10,8 \pm 4,5$ vs 10,2 $\pm 1,5)$.
El contenido medio de GC en los diferentes órganos de cobayos se presentan en la figura 2. Se observa un menor contenido de este marcador en corazón $(24,7 \pm 20,8$ vs $15,7 \pm 6,4)$, riñones $(73,2 \pm 47,3$ vs $53,2 \pm 25,3)$ y en testículos $(60,9 \pm 8,1$ vs $33,6 \pm 13,4)$ de cobayos de altura, aunque solamente las gónadas presentan una diferencia significativa $(p=o, o o o)$ frente a los del nivel del mar. Puede observarse también que, aunque en la altura el promedio en hígado (24,6 $\pm 15,1$ vs $28,3 \pm 10,9)$ y pulmones $(50,7 \pm 7,8$ vs $69,3 \pm 29,2)$ son mayores, estas diferencias no tienen significación estadística.

El nivel medio de TT en órganos de cobayos oriundos de altura fue menor en corazón (124, $6 \pm$ $10,2$ vs $96,0 \pm 9,3 ; p=o, o o o)$ y pulmones $(108,9 \pm 27,0$ vs $79,8 \pm 4,6 ; p=0,003)$, mientras que en riñones el nivel medio de grupo tioles fue mayor $(53,0 \pm 9,8 \mathrm{vs}$ $75,4 \pm 9,9 ; p=o, o o o$ ) comparado con los cobayos que habitan a nivel del mar. En hígado $(86,9 \pm 31,1$ vs 10,0 $\pm 14,4)$ y testículos $(144,6 \pm 35, \mathrm{o}$ vs $116,5 \pm 57,8)$ no se evidenciaron diferencias significativas (figura 3 ).

También se evaluó el nivel de asociación entre los niveles de hidroperóxidos de proteínas, grupos carbonilos y tioles totales en los diversos órganos de cobayos. No se encontró correlación entre los parámetros estudiados.

\section{DISCUSIÓN}

La vida en las grandes alturas está relacionada con mecanismos de adaptación bioquímicos que se hacen posibles mediante el control y la regulación metabólicas como respuesta no solamente a la hipoxia, sino también a los cambios de temperatura, radiación, etc. Estas adaptaciones, que ocurren en diferentes niveles de organización en el organismo, son necesarias para mantener la homeostasis y mantener así la condición fisiológica del organismo.

La hipoxia de las grandes alturas está relacionada con un aumento de las EROS, numerosos estudios demuestran un incremento en la producción de indicadores de estrés oxidativo en músculo, sangre, orina y en diferentes tejidos en respuesta a la hipoxia aguda ${ }^{(1,2)}$. Estudios en nativos de las grandes alturas evidencian que, en la hipoxia crónica también hay un incremento en la formación de EROS ${ }^{(3)}$.

Existen escasos estudios relacionados al metabolismo oxidativo de las proteínas. Las proteínas son los componentes orgánicos más abundantes de los organismos vivos y se sabe que reaccionan ávidamente con EROS de significancia biológica ${ }^{(10)}$. Por otra 
parte, las proteínas son posiblemente el vehículo más inmediato para originar daño oxidativo en las células, ya que a menudo son catalizadores en vez de mediadoras; por lo tanto, el efecto del daño a una molécula es mucho mayor ${ }^{(6)}$.

El presente estudio se realizó en órganos de cobayos que habitan en las grandes alturas $(3660 \mathrm{~m})$, como a nivel del mar (150 $\mathrm{m})$. Estos animales están perfectamente adaptados a su propio medio ambiente.

En el presente estudio se encontraron altas concentraciones de $\mathrm{POOH}$ en hígado y testículos de cobayos de grandes alturas. En el primer caso, se conoce que el hígado es particularmente sensible al estrés oxidativo inducido por la hipoxia y que presenta una menor actividad de glutation peroxidasa (11), el alto contenido de ácido glutámico, ácido aspártico, leucina y prolina del hígado, aumenta su vulnerabilidad a sufrir el proceso de peroxidación proteica, posiblemente en estos aminoácidos ${ }^{(12)}$.

En relación a los testículos, es bien conocida la susceptibilidad de las células somáticas y germinales testiculares ante un proceso de estrés oxidativo por efecto de la hipoxia ${ }^{(13)}$, posiblemente estará acompañado por una disminución en la expresión de la actividad enzimática de $\mathrm{Cu}$ / Zn SOD citosólico y Mn SOD, la principal línea de defensa antioxidante presente en células germinales ${ }^{(14)}$.

Estudios en corazón de ratas bajo condiciones anaerobias, muestran una

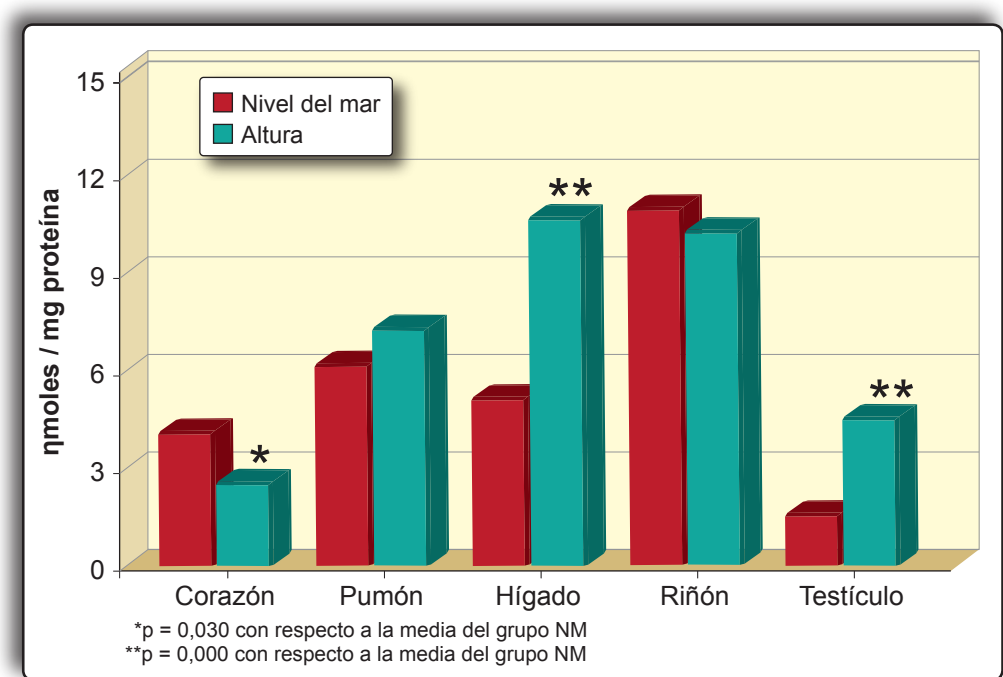

Figura 1. Niveles medios de hidroperóxido de proteínas (†moles/mg proteína) en órganos de cobayos.

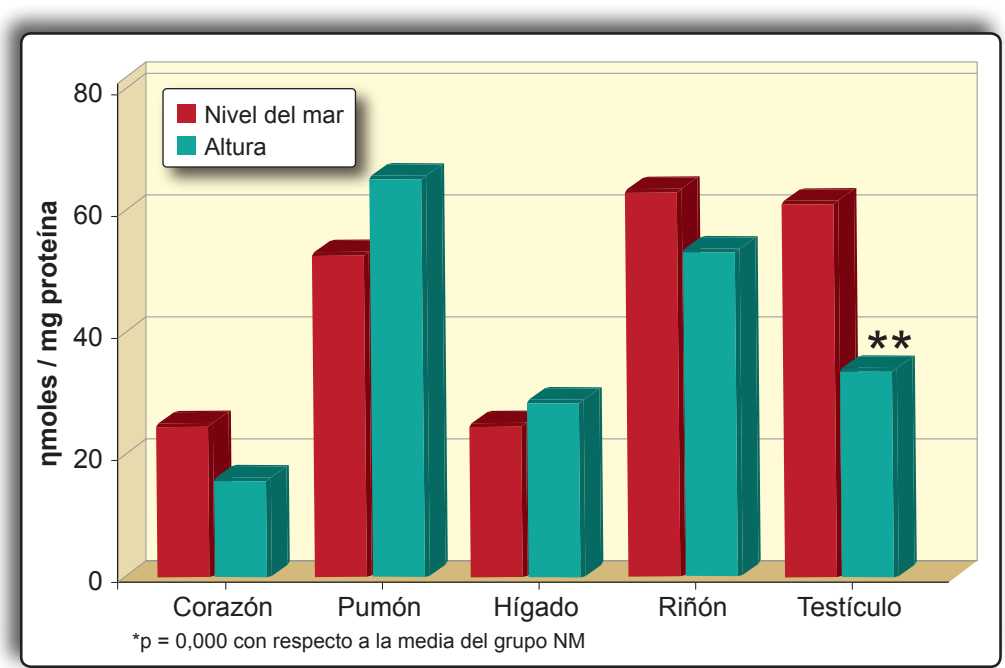

Figura 2. Niveles medios de grupos carbonilo ( $\eta$ moles/mg proteína) en órganos de cobayos. inhibición de síntesis de proteínas y de miosina. Esta inhibición parece ser a un bloqueo en la incorporación de aminoácidos libres del pool intracelular hacia la proteína ${ }^{(15)}$, tal vez sea la explicación por la que se encontraron bajas concentraciones de hidroperóxidos de proteínas en corazón de cobayos de altura.

No se encontró diferencia significativa en el contenido de GC en corazón, pulmones, hígado y riñones. La baja concentración de grupos carbonilo (GC) en testículos de cobayos nativos de altura indicaría una disminución en la formación de EROS, aunque diversos estudios han demostrado que en las grandes alturas existe un descenso en la velocidad de la síntesis proteica, por lo que el bajo nivel de GC podría justificarse por un aumento de la concentración de $\mathrm{Ca}^{+2}$ intracelular, el cual activaría a las enzimas proteasas dependientes de calcio -que atacan al citoesqueletohasta degradar las proteínas dañadas por las EROS (16).

En corazón, pulmones y testículos de cobayos nativos de altura, se encontró una menor concentración de tioles totales con respecto a los cobayos que habitan a nivel del mar. El papel antioxidante de los tioles totales, donde el glutatión reducido (GSH) es el principal componente, es prevenir el daño oxidativo a las proteínas. El GSH es un importante antioxidante endógeno que puede neutralizar EROS, mantener el equilibrio redox intracelular y proteger los tejidos del estrés oxidativo; además, mantiene a los antioxidantes exógenos, vitaminas $\mathrm{C}$ y $\mathrm{E}$, en sus formas reducidas. Los resultados obtenidos nos permiten interpretar que hay 


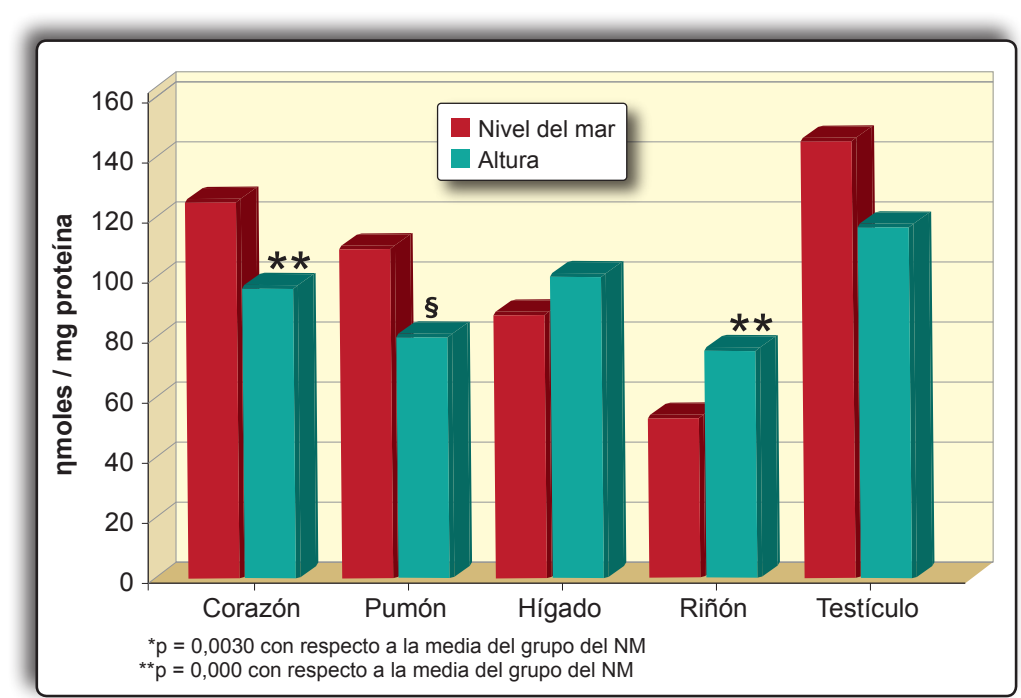

Figura 3. Niveles medios de tioles totales (†moles/mg proteína) en órganos de cobayos.

un mayor consumo de este péptido, principalmente en corazón y pulmones.

Se encontró una elevada concentración de tioles totales en riñones de cobayos de altura. En el residente de altura, la función renal (filtración glomerular y flujo plasmático) se encuentra significativamente disminuida, anteello, posiblementeel organismoactive el sistema tiol, incrementándose la concentración del grupo tioles, evitando así se desencadene un desequilibrio por las condiciones fisiológicas existentes a nivel renal ${ }^{(1)}$. El glutatión protege a nivel tubular, parece ser el mecanismo principal en células tubulares para mantener al glutatión intracelular en estado reducido. Este antioxidante inhibe las reacciones que desencadenan las especies reactivas de oxígeno y es sensible al nivel de vitamina $C$ en el medio intracelular, cuya disminución dispara una señal metabólica que lleva a incrementar la síntesis de glutatión ${ }^{(17)}$.

Por otro lado, está demostrado que la hipoxia desmejora la capacidad de los sistemas antioxidantes. Diversos estudios refieren que en las grandes alturas se ve reducida la actividad y el contenido de ciertas enzimas antioxidantes ${ }^{(18)}$. Una alteración en el estado redox tiólico celular puede llegar a ser potencialmente desencadenante de alteraciones fisiopatológicas en el ser humano.

\section{CONCLUSIONES}

El análisis de los resultados obtenidos en el estudio de oxidación de proteínas en órganos de cobayos nativos de altura (Huancavelica, $3660 \mathrm{msnm}$ ), permite concluir que, la hipoxia hipobárica influye en el proceso de oxidación de proteínas en forma selectiva, lo cual se evidencia por las mayores concentraciones de $\mathrm{POOH}$ y GC en algunos órganos de cobayos de altura comparados con los del nivel del mar y que la respuesta antioxidante endógena, representada por los niveles de los tioles totales, sigue el mismo patrón. Se requiere estudiar otros biomarcadores de oxidación de proteínas, tales como 3-nitrotirosina y productos de oxidación avanzada de proteínas (AOPP), así como otros antioxidantes para poder comprender el papel de la hipoxia en la oxidación proteica.

\section{REFERENCIAS BIBLIOGRÁFICAS}

1. Askey EW. Word at high altitude and oxidative stress: antioxidant nutrients. Toxicology 2002; 180(2): 107-19.

2. Sinha S, Ray US, Tomar OS, Singh SN. Different adaptation patterns of antioxidant system in natives and sojourners at high altitude. Respir Physiol Neurobiol 2009; 167(3): 255-6o.

3. Jefferson JA, Simoni J, Escudero E, et al. Increased oxidative stress following acute and chronic high altitude exposure. High Alt Med Biol 2004;5(1):61-69

4. Skvarilová M, Bulava A, Stejskal D, Adamovská S, Bartek J. Increased level of advanced oxidation products (AOPP) as a marker of oxidative stress in patients with acute coronary syndrome. Biomed Pap Med Fac Univ Palacky Olomouc Czech Repub 2005 149(1): 83-7.

5. Liu J. Protein hydroperoxides $(\mathrm{POOH})$. The University of Iowa. Iowa, 2003. [En línea] Último acceso 10 de marzo 2014. Disponible en http://www.healthcare.uiowa.edu/ corefacilities/esr/education/2003/1/LiuJingru-paper-1.pdf

6. Dalle-Donne I, Aldini G, et al. Protein carbonylation, cellular dysfunction, and disease progression. J Cell Mol Med 2006; 10(2): 389-406.

7. Nourooz Z, Sarmadi T. Mesurement of plasma hydroperoxides concentration by ferrous oxidation of xylenol orange assay in conjunction with triphenylphosphine. Anal Biochem 1994; 220(2): 403-9.

8. Levine RL, Garland D, Oliver CN, Amici A, Climent I, Lenz AG, et al. Determination of carbonyl content in oxidatively modified proteins. Methods Enzymol 1990; 186: 464-78.

9. Cakatay U, Telci A, Kayali R, Tekeli F, Akçay T, Sivas A. Relation of aging with oxidative protein damage parameters in the rat skeletal muscle. Clinical Biochemistry 2003; 36(1): 51-5.

10. Du J, Gebicki JM. Proteins are major initial cell targets of hydroxyl free radicals. Int J Biochem Cell Biol 2004; 36(11): 2334-43. 
11. Nakanishi K, Tajima F, Nakamura A, Yagura S, Ookawara $\mathrm{T}$, Yamashita $\mathrm{H}$, et al. Effects of hypobaric hypoxia on antioxidant enzymes in rats. J Physiol 1995; 489: 869-76.

12. Radák Z, Nakamura A, Nakamoto H, Asano K, Ohno $\mathrm{H}$, Goto $\mathrm{S}$. A period of anaerobic exercise increases the accumulation of reactive carbonyl derivates in the lungs of rats. Pflugers Arch 1998; 435(3):439-41.

13. Farias JG, Puebla M, Acevedo A, Tapia P, Gutiérrez E, Zepeda A, et al. Oxidative stress in testis and epididymis under intermittent hypobaric hypoxia in rats: Protective role of ascorbate supplementation. J Androl 2010; 31(3): 314-21.

14. Bauché F, Fouchard MH, Jégou B. Antioxidant system in rat testicular cells. FEBS Lett 1994; 349(3): 392-6.

15. Bigard X, Sanchez H, Birot O, Serrurier B. Myosin heavy chain composition of skeletal muscles in young rats growing under hypobaric hypoxia conditions. J Appl Physiol 200o; 88(2): 479-486.
16. Ono Y, Sorimachi H. Calpains: an elaborate proteolytic system. Biochim Biophys Acta 2012; 1824(1):224-36

17. Visarius TM, Putt DA, Schare JM, Pegouske DM, Lash LH. Pathways of gluthatione metabolism and transport in isolated proximal tubular cells from rat kidney. Biochemical Pharmacology 1996; 52(2): 259-72.

18. Bakonyi T, Radak Z. High altitude and free radicals. Journal of Sports Science and Medicine 2004; 3(2): 64-9.

\section{Correspondencia:}

Nombre: $\quad$ Elizabeth Carranza Alva

Dirección: Instituto Nacional de Biología Andina Facultad de Medicina - UNMSM.

E-mail: $\quad$ ecarranzalva@gmail.com 